\title{
IN-SITU CHEMICAL AND ISOTOPIC MEASUREMENTS OF THE ATMOSPHERE OF JUPITER
}

\author{
P.R. MAHAFFY \\ Goddard Space Flight Center \\ Greenbelt, $M D 20771$ USA \\ S.K. ATREYA \\ University of Michigan \\ Department of Atmospheric, Oceanic and Space Sciences \\ 2455 Hayward Street, Ann Arbor, MI 48109 USA \\ H.B. NIEMANN \\ Goddard Space Flight Center \\ Greenbelt, MD 20771 USA \\ AND \\ T.C. OWEN \\ University of Hawaii \\ Institute for Astronomy \\ 2680 Woodlawn Drive, Honolulu, HI 96822 USA
}

\begin{abstract}
Insights into both the detailed composition of Jupiter's atmosphere and unexpected local meteorological phenomena were revealed by in-situ measurements from the Galileo Probe Neutral Mass Spectrometer taken on December 7, 1995. Measurements of the neutral atmospheric composition from a pressure of 0.5 bar to approximately 21 bar revealed the mixing ratios of the major species helium and hydrogen as well as numerous minor constituents including methane, water, ammonia, ethane, ethylene, propane, hydrogen sulfide, neon, argon, krypton, and xenon. This instrument measured the isotope ratios ${ }^{3} \mathrm{He} /{ }^{4} \mathrm{He}, \mathrm{D} / \mathrm{H}$, and ${ }^{13} \mathrm{C} /{ }^{12} \mathrm{C}$ as well as the isotopes of neon, argon, krypton, and xenon. A summary is given of progress that has been made in refining preliminary estimates of the abundances of condensable volatiles and noble gases as a result of an ongoing laboratory study using a nearly identical engineering unit. The depletion of simple condensable species to depths well below their expected condensation levels is explained by a local downdraft in the region of the probe entry. The mass spectrometer data suggests that different species may recover at different depths and this may be due to lateral mixing of Jovian air.
\end{abstract}

\section{Introduction}

The measurements described took place on December 7, 1995 as the Galileo Probe following its parachute deployment descended into the atmosphere of Jupiter. It had been anticipated prior to this encounter that measurements of the Jovian chemical and isotopic composition by the Galileo Probe Neutral Mass Spectrometer (GPMS) at depths of 5 to 20 bar would provide a direct comparison with solar abundances for elements such as $\mathrm{He}, \mathrm{O}, \mathrm{N}, \mathrm{S}, \mathrm{C}$ and the noble gases. This together with the measurement of key isotopes was in turn expected to enable a better understanding of mechanisms of formation of the planet through direct capture of nebular gas or infusion of icy planetesimals.

However, surprisingly, the probe entered a relatively cloud-free region, a 5- $\mu \mathrm{m}$ " hotspot," which was atypical of the entry sites at that latitude. Species such as $\mathrm{H}_{2} \mathrm{O}, \mathrm{H}_{2} \mathrm{~S}$, and $\mathrm{NH}_{3}$ were substantially depleted in the early and mid portion of the descent from their predicted average atmospheric levels. Following a brief description of the experiment and the post-encounter calibration activities 


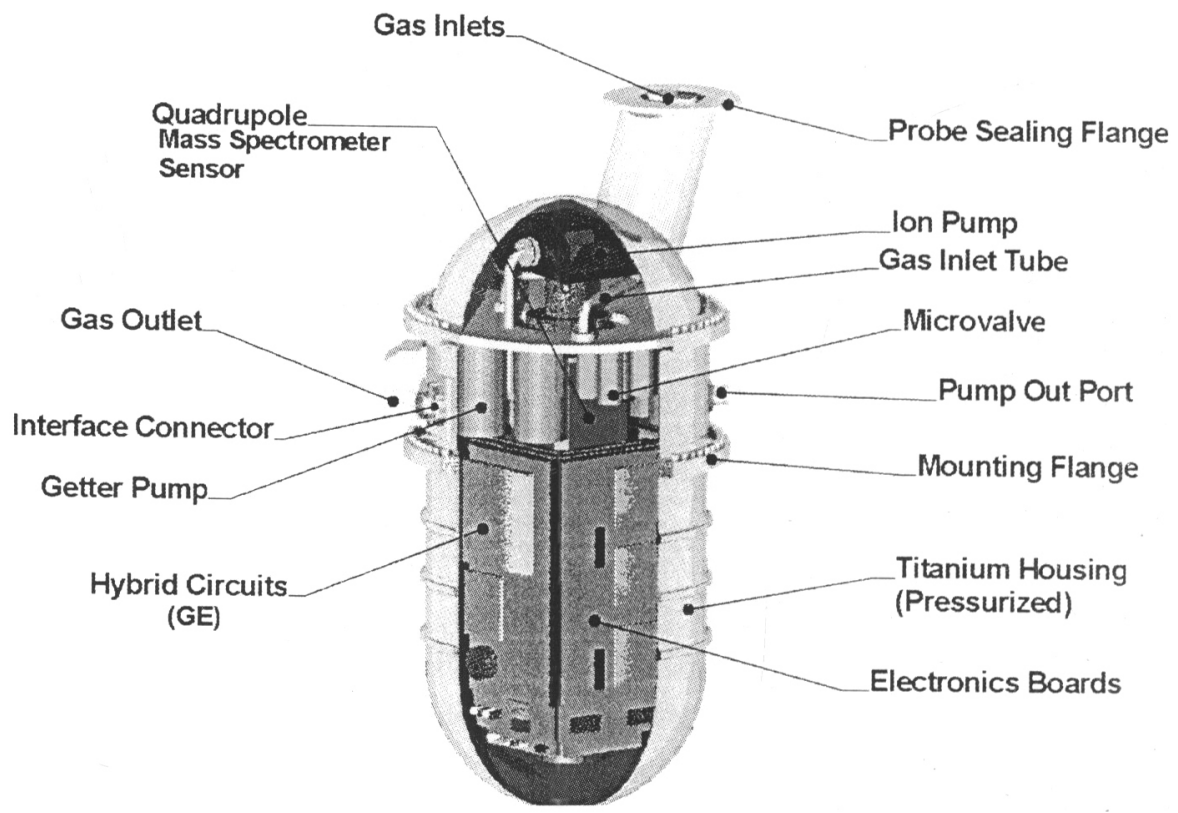

Figure 1. An illustration of the GPMS FU. All of the electronics for the instrument were wrapped around the analyzer, while the pumps and valves are all mounted on a gas manifold plate.

in the next section, we give a summary of the noble-gas and condensable volatile findings from the GPMS. We describe progress that has been made to date for this portion of the data set based both on further analysis and on continuing post-encounter laboratory calibrations. Finally, before concluding we discuss the implications of the strong depletion of volatiles discovered by the GPMS and other measurements.

\section{Mass Spectrometer Experiment}

An illustration of various elements of the GPMS Flight Unit described in detail by Niemann et al. (1992) are shown in Fig. 1 and a picture of just the vacuum hardware in Fig. 2. The instrument was built around a quadrupole analyzer mass spectrometer with a mass range 2 to $150 \mathrm{amu}$ whose ion source employed a magnetically confined electron beam emitted from a hot filament, and whose ion detector was an electron multiplier operated in a pulse counting mode. The ion source and analyzer volumes were continuously pumped by a miniature sputter ion pump and by chemical getters. The gas-processing system allowed for several modes of instrument operation. Measurements of gas introduced to the ion source from one of two direct leaks (DL1 or DL2) or of gas thermally released from enrichment cells (EC1 or EC2) loaded during an earlier time during the descent could be introduced into the ionization region of the mass spectrometer by appropriate selection of valve states. The operation of one of the enrichment cells was optimized for the detection of rare gases (RG). Several background spectra were taken during the course of the descent as were several higher-resolution (1/8 amu) mass scans to verify instrument tuning. The instrument implemented a descent measurement sequence through its various modes which had been programmed before launch. Typically, masses were not simply scanned sequentially, but a higher number of integration periods were spent on those mass values of special interest such as the regions where the noble gases contributed to the signal.

The GPMS ion detector employed fixed 1/2-second integration periods for each $\mathrm{m} / \mathrm{z}$ value sampled. These counts for the entire descent-selected masses are plotted in Fig. 3, illustrating the signals whose counts come primarily from the condensible species $\mathrm{H}_{2} \mathrm{~S}$ at 34 amu and $\mathrm{H}_{2} \mathrm{O}$ at 18 amu compared to the signals from methane fragments at $14 \mathrm{amu}, \mathrm{H}_{2}$ at $2 \mathrm{amu}$, and ${ }^{4} \mathrm{He}$ at $4 \mathrm{amu}$. 


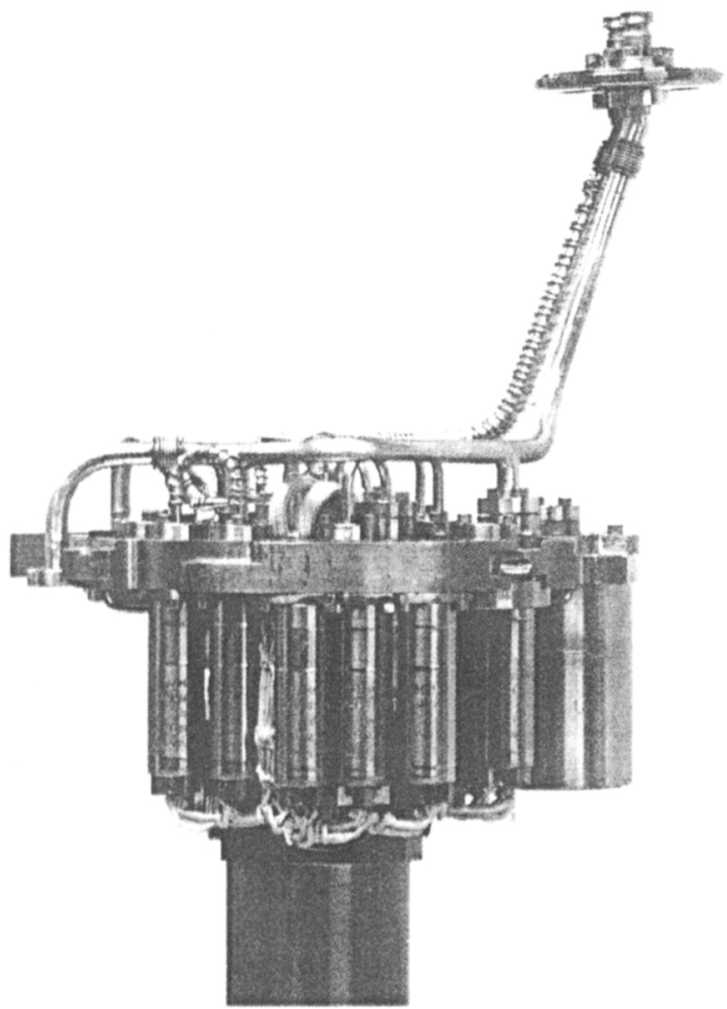

Figure 2. A photograph of the gas inlet system and the GPMS sensor. The larger cylinders on the inlet plate are getter pumps.

The mixing ratios of the latter three species are not expected to change over the course of the probe descent. Figure 4 similarly illustrates the counts from the noble gases helium, argon, and xenon. Just before signal was lost from the Probe, the detector counts went to zero and the last integration period where non-zero counts were measured was integration step \#6851. A polynomial extrapolation between the individual measurements for each of the 2- to 150-amu mass values allows full spectra to be extracted at any step in the descent sequence. Such a spectrum is illustrated in Fig. 5 at step number 4250 , which corresponds to an atmospheric pressure of 11.37 bars. Several of the species identified in the atmosphere are labeled in this spectrum.

\section{Calibrations of the Flight Unit and the Engineering Unit}

To obtain atmospheric mixing ratios from spectra such as illustrated in Fig. 5, calibration for each species of interest is required. The response of the GPMS to each individual species is a function of the ion-source density for that species, the ionization cross section at the electron energy selected into the parent molecular ion or fragment of interest, and the mass-spectrometer transmission and detector efficiency for each ion. The source density, in turn, is dependent on the system pumping characteristics for that species, the capillary inlet flow characteristics for that inlet pressure, and in some cases, instrument-produced background sources of the species of interest. The highly miniaturized pumping system developed for the GPMS incorporates both passive chemical getter pumps and an ion pump resulting in a wide range of pumping characteristics for different chemical species. For example, hydrogen and nitrogen are pumped well by the chemical getters while the noble gases and methane are only pumped by the ion pump.

The same calibration system which had been used in 1985 to establish the response of the 


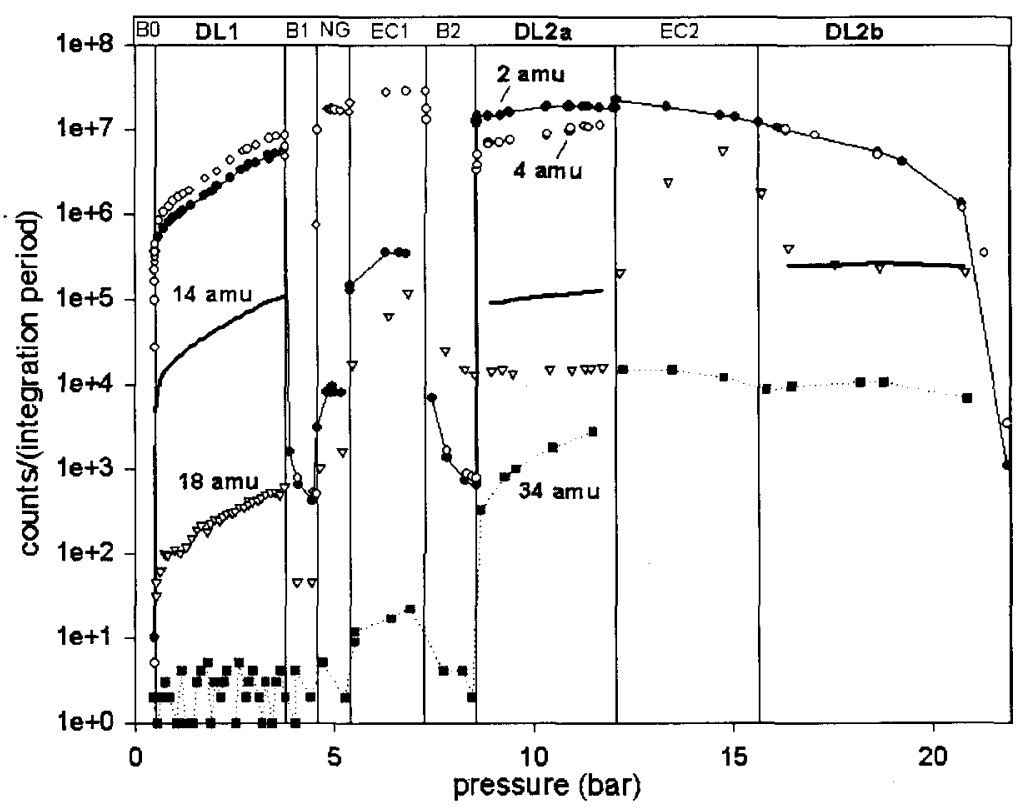

Figure 3. Counts/integration period are show for the several mass values listed in the figure. Direct sampling of the atmosphere occurred during the DL1 and DL2a and DL2b time periods using two separate inlet systems. Instrument background measurements were obtained during the $\mathrm{B} 0, \mathrm{~B} 1$, and $\mathrm{B} 2$ periods while NG designates the noble gas experiment, where active gases had been removed from a small volume with a chemical pump, leaving primarily the noble gases together with methane. Gases thermally released from enrichment cells were introduced to the ion source during the periods labeled EC1 and EC2. The 4-amu and 2-amu counts at times following the initiation of the EC2 experiment saturate the detector and thus a significant fraction of the ions reaching the detector are not counted.

GPMS Flight Unit (FU) to various gases is presently operational to carry out related studies on a GPMS Engineering Unit (EU) for conditions and species not studied with the FU. In the EU all elements including the pumps, the enrichment cells, the ion source, the analyzer, and the detector resemble those on the FU. Differences between the FU and EU with regard to inlet leak conductance are described below. The calibration system enables gas mixtures to be created and circulated through the inlet lines of the GPMS unit at pressures from millibars to 22 bars. Wide dynamic range pressure gauges together with known gas expansion volumes allow mixing ratios down to sub-parts-per-billion to be generated in mixtures which represent the Jovian atmosphere with respect to major and significant minor species. Presently, gases from up to six different tanks can be mixed into the circulation system.

The CAMAC-based data acquisition system allows flexibility in control of the GPMS EU voltages and in data display. Ion and electron focusing lens voltages used in the EU are very close to those used in the FU with small adjustments made to account for differences in geometry between the ion optical subsystems of the two units. The GPMS EU is operated with a flight spare RF board to duplicate as closely as possible the behavior of the mass analyzer.

The GPMS design employed four separate micron-sized inlet capillary leaks. Two of these directly sampled the atmospheric gas flowing through the inlet system and two allowed the gas released from the enrichment cells to be introduced into the ion source. The former measurements took place during the time periods labeled DL1 and DL2a and DL2b in Figs. 3 and 4 and the latter during the measurement periods labeled NG, EC1, or EC2. Presently, the EU has been refurbished from its 1985 condition to replace the enrichment cell leaks with those having nearly the same gas conductance as the FU. This has allowed EU calibration activities at the same trace levels of krypton and xenon present at Jupiter to be initiated using the EU enrichment cells. However, for all DL2 EU studies reported to date, the conductance of the leak in the EU was more than a factor of two smaller than the FU. This means that ion source densities equivalent to those realized in the 12- to 22-bar region of the descent are not yet achieved in the EU. Conclusions based on 


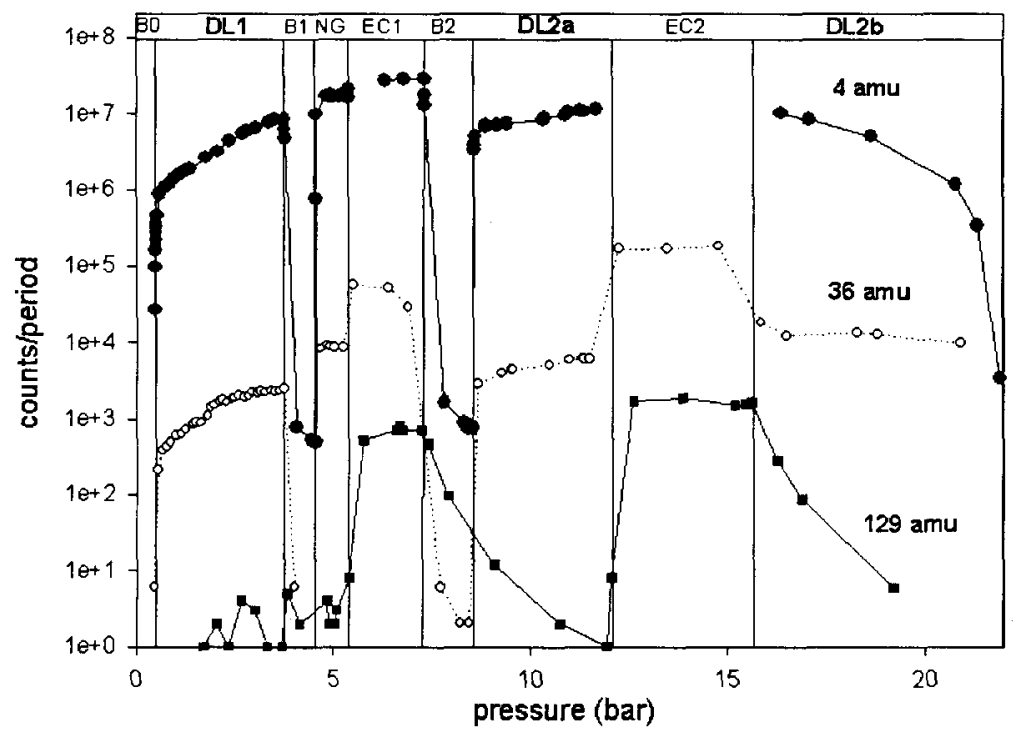

Figure 4. A mass spectrum at the 11.37-bar region of the atmosphere is shown where possible skew in the spectrum due to the sampling has been removed by fitting the counts per integration period at each mass-to-charge $(\mathrm{m} / \mathrm{z})$ value to a polynomial and taking the resulting spectrum at integration period \#4250.

counts measured in the DL2b region must be considered tentative until this refurbishment and these studies have been carried out.

\section{Overview of GPMS Measurements of the Noble Gases, $\mathrm{H}_{2} \mathrm{~S}$, and $\mathrm{H}_{2} \mathrm{O}$}

A preliminary report (Niemann et al. 1996) made soon after the encounter gave a list of the species detected in Jupiter's atmosphere, their mixing ratios, and selected isotopic abundances. Major findings included a confirmation of the helium atmospheric depletion relative to the predicted protosolar hydrogen, a substantial depletion in the atmospheric neon relative to the solar value, and an argon mixing ratio which was slightly greater than solar. The helium mixing ratio to hydrogen of $0.156+0.006$ derived from the GPMS 4-amu to 2-amu count ratio was nearly identical to the value independently reported by the team analyzing data from the Helium Abundance Detector (von Zahn and Hunten 1996) also on the Probe. The ${ }^{20} \mathrm{Ne}$ mixing ratio was determined by the GPMS to be approximately $0.1 \times$ solar. The depletion of the ${ }^{4} \mathrm{He}$ relative to the value modeled for the protosolar value, although less than that derived from the Voyager Flyby observations (Conrath et al. 1984) was nevertheless significant and consistent with the predicted differentiation of helium droplets from metallic hydrogen (Stevenson et al. 1977) deep in the interior of Jupiter. It had been predicted prior to the encounter (Roulston et al. 1995) that neon could be miscible in the liquid helium phase precipitating out deep in the interior and its abundance in the outer atmosphere could be depleted. The low value of the neon to hydrogen ratio relative to the solar ratio measured by the GPMS is consistent with this model.

Very high limits on the mixing ratios for the heavy noble gases were given in a preliminary report (Niemann et al. 1996). These were $<50 \times$ solar for xenon and $<5 \times$ solar for krypton. These can both now be reduced considerably based on the preliminary studies carried out on the EU enrichment cell system. For example for xenon the present limit $R_{\mathrm{Xe}}$ of the Jovian xenon mixing ratio relative to hydrogen is estimated to be $0.3 R S_{\mathrm{Xe}}<R_{\mathrm{Xe}}<3 R S_{\mathrm{Xe}}$ (where $R S_{\mathrm{Xe}}$ is the corresponding solar value) with further refinements anticipated as this work continues. The primary contribution to the DL2a count rates on which the earlier estimates were based now appear to be residual instrument background counts from heavy noble gases and produced from the enrichment cells. The decay of the 129-amu signal from xenon following the closure of the valve to the ion source from the enrichment cell is shown in Fig. 4 and supports this conclusion. The 129-amu signal does 


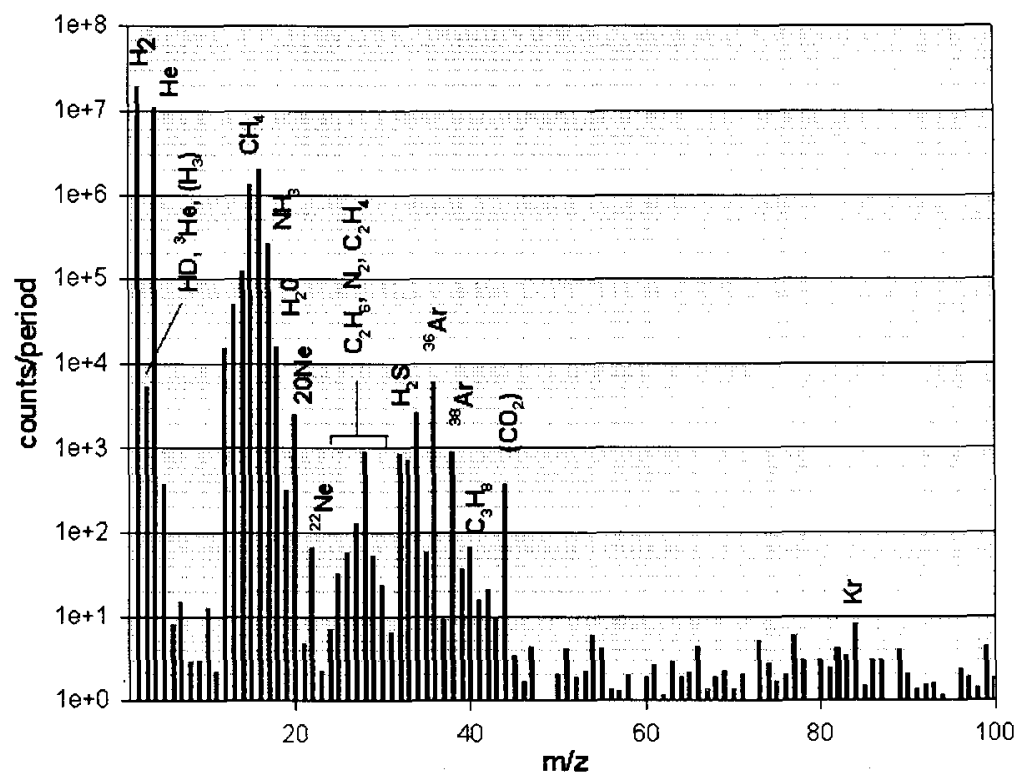

Figure 5. Counts/integration period are shown for 4, 36, and $129 \mathrm{amu}$. Contributions to these signals come primarily from ${ }^{4} \mathrm{He},{ }^{36} \mathrm{Ar}$, and ${ }^{129} \mathrm{Xe}$. Use of the enrichment cells enabled measurements of the krypton and xenon abundance and isotopic fractionation.

not increase when the DL2a measurement sequence begins at 8.5 bars, but simply continues its exponential-like decay from the enrichment cell value.

The raw 18- and 34-amu counts are given in Fig. 3 and compared with the signal from noncondensible species in the atmosphere. The primary contribution to the 18-amu signal is water with a small contribution from doubly ionized ${ }^{36} \mathrm{Ar}$. The primary contribution to the 24-amu signal is $\mathrm{H}_{2} \mathrm{~S}$. The trend in the $\mathrm{H}_{2} \mathrm{~S}$ and $\mathrm{H}_{2} \mathrm{O}$ counts from the DL2a to the DL2b region shows a dramatic increase at both of these mases, but with an apparently larger jump in the water abundance across the EC2 region where the direct atmospheric measurements were masked by the signal from the enrichment cell EC2. As discussed above, instrument calibrations in the DL2b region have not yet been carried out. The trends nevertheless suggest a dramatic recovery in the abundance of both of these species in the 10- to 20-bar region of the atmosphere.

\section{5. $\mathrm{H}_{2} \mathrm{O}, \mathrm{H}_{2} \mathrm{~S}$ and $\mathrm{NH}_{3}$ : Implications for Local Meteorology}

Although the $\mathrm{H}_{2} \mathrm{~S}$ and $\mathrm{H}_{2} \mathrm{O}$ measured by GPMS, and $\mathrm{NH}_{3}$ measured by other instruments in the Galileo probe entry region are all greatly depleted to depths well below their expected condensation levels, their mixing ratios increase gradually with depth and perhaps eventually even recover to their normal deep atmospheric abundances. Before the Galileo probe measurements, only the existence of the topmost cloud of ammonia-ice had been confirmed, although each of these gases is expected to condense in the Jovian atmosphere. This condensation is expected to be either direct or by thermochemistry.

For example, $\mathrm{H}_{2} \mathrm{~S}$ itself does not condense at the prevalent temperatures but its reaction with $\mathrm{NH}_{3}$ produces ammonium hydrosulfide $\left(\mathrm{NH}_{4} \mathrm{SH}\right)$ or, as proposed by Owen and Mason (1969), ammonium sulfide $\left(\mathrm{NH}_{4}\right)_{2} \mathrm{~S}$, which does solidify at the these temperatures. Although its expected condensation level lies well above 2.2 bars for solar S/H and 2.7 bars for $3 \times$ solar S/H (Atreya and Romani 1985; Weidenschilling and Lewis 1973), the GPMS upper limit on the $\mathrm{H}_{2} \mathrm{~S}$ mixing ratio at $p<4$ bar is $10^{-6}$, or $0.03 \times$ solar. Larson et al. (1984) had noted a depletion in $\mathrm{H}_{2} \mathrm{~S}$ down to the 0.8-bar level from ground-based 2.7-micron observations. This is not surprising since $\mathrm{H}_{2} \mathrm{~S}$ is expected to be removed by condensation deeper in the atmosphere.

The abundance of $\mathrm{H}_{2} \mathrm{~S}$ in the 4- to 8-bar region was below the sensitivity limit of the GPMS. 
The $\mathrm{H}_{2} \mathrm{~S}$ mixing ratio gradually rises in the 8- to 20-bar region from $0.3 \times$ solar at 8 bars to nearly $2.7 \times$ solar at approximately 15 bars. At this last value there is an indication that $\mathrm{H}_{2} \mathrm{~S}$ has leveled off but this conclusion should be interpreted with caution until calibrations with the refurbished EU described above have been completed. It is nevertheless evident that there is depletion of $\mathrm{H}_{2} \mathrm{~S}$ to depths well below its expected condensation level and that it gradually builds up in its mixing ratio with depth toward its deep atmospheric value.

Water vapor, like $\mathrm{H}_{2} \mathrm{~S}$, is found to be greatly depleted to depths well below its expected condensation levels of $5.4-6.5$ bars for solar and $2 \times$ solar O/H (Atreya and Romani 1985; Weidenschilling and Lewis 1973). As illustrated in Fig. 3, the increase between 10 and 20 bars may be on the order of a factor of 10 if unexpected instrument effects do not play a role in changing the count rates for this ion over this pressure range. The upper limit from the GPMS analysis for pressures of 4 bars or less is $10^{-6}$. This corresponds to $1 \%$ saturation at 4 bars. Data from Galileo Orbiter's 5-micron NIMS observations are consistent with the GPMS data (Carlson et al. 1996, 1997; Roos-Serote et al. 1996) since they also point to low values of water vapor in this part of the atmosphere and even down to 10-bar pressure.

The GPMS FU ammonia data show large transients, perhaps due partially to enrichment cell memory transients of the kind illustrated for the xenon data. However, the Net Flux Radiometer data can be best interpreted with $\mathrm{NH}_{3}$ between 1 to $2 \times$ solar at $3-6$ bars (Sromovsky et al. 1996). The mixing ratio of $\mathrm{NH}_{3}$ must drop by a factor of 20 from 3 to 1 bar, or to $5-10 \%$ of solar, and by another factor of 4 , i.e. to $1-2 \%$ of solar between 1 and 0.5 bars (Sromovsky et al. 1996). The clouds are not expected to form before reaching approximately 0.7 bar for solar N/H (Atreya and Romani 1985; Weidenschilling and Lewis 1973). NIMS observations (Carlson et al. 1996, 1997) also confirm the depletion in ammonia at low pressure. Analysis of the VLA detection of the Probe's signal (W. Folkner, personal communication, 1997) also confirms the trend of increasing mixing ratio with depth in ammonia and, in fact, may point to a greater depth at which $\mathrm{NH}_{3}$ recovers to its normal deep atmospheric value than implied by the NFR data.

The behavior of the condensible volatiles with depth along the Probe's trajectory can best be explained in terms of local meteorology. It has been proposed (Atreya et al. 1996, 1997; Owen et al. 1996,1997$)$ that the probe entry region represents a downdraft where condensible volatiles are absent. This takes place in an adjacent updraft cell first by condensation and then by sedimentation preceded by particle growth due to coagulation and coalescence. If the the air mass rises above the lifting condensation levels the mixing ratios are limited even further. In fact, at Jupiter's $110 \mathrm{~K}$ tropopause, the air would be nearly all dried out of its condensible volatiles. The descent of this airmass as a downdraft into the probe entry region would enable mixing with the probe-region air and a gradual recovery with depth in the condensible volatile mixing ratios. This is precisely what has been observed. Showman and Ingersoll (1996) propose a thermodynamic heat engine in reverse, a refrigerator, to drive this downdraft and keep it dry to such great depths. Possible horizontal or lateral mixing of the normal Jovian air into the probe entry region (Atreya et al. 1997) may allow condensible volatiles to recover at different depths.

The measured abundances of the condensibles explain the nearly cloud-free trajectory of the Probe (Ragent et al. 1996). In fact, the concentrations of the very tenuous clouds at 0.5 bar and 1.34 bar and the lack of a water cloud (Ragent et al. 1996) are entirely consistent with an $\mathrm{NH}_{3}$-ice cloud at 0.5 bar and an $\mathrm{NH}_{4} \mathrm{SH}$-solid cloud at 1.34 bar (Atreya et al. 1997) in view of the depleted levels of the condensibles volatiles measured on the Probe.

\section{References}

Atreya, S.K., and Romani, P.N. (1985) In: Planetary Meteorology, G.E. Hunt (Ed.), Cambridge University Press, pp. $17-68$.

Atreya, S.K. et al. (1996) Bull. Am. Astron. Soc., 28, No. 3, p. 1133.

Atreya, S.K. et al. (1997) In: Three Galileos: The Man, The Spacecraft, The Telescope, J. Rahe, C. Barbieri, T. Johnson, A. Sohus (Eds.), Kluwer Academic Press, in press.

Carlson, R. et al. (1996) Science, 274, pp. 385-388.

Carlson, R. et al. (1997) Near IR spectroscopy of the atmosphere of Jupiter. In: this volume.

Conrath, B.J. et al. (1984), Astrophys. J., 282, pp. 807-815 (this paper also describes the Jupiter results from Voyager).

Larson, H.P. et al. (1984) Icarus, 60, pp. 621-639.

Owen, T.C. and Mason, H.P. (1969) J. Atmos. Sci., 26, pp. 870-873.

Niemann, H.B. et al. (1992) Space Sci. Rev., 60, p. 168. 
Owen, T.C. et al. (1996) EOS, Trans. Suppl., 77, 46, p. F438.

Owen, T.C. et al. (1997) In: Three Galileos: The Man, The Spacecraft, The Telescope, J. Rahe, C. Barbieri, T. Johnson, A. Sohus (Eds.), Kluwer Academic Press, in press.

Ragent, B. et al. (1996) Science, 272, pp. 854-855.

Roos-Serote, M. et al. (1996) Bull. Am. Astron. Soc., 28, No. 3, p. 1135.

Roulston, M.S. and Stevenson, D.J. (1995) EOS, 76, p. 343.

Showman, A.P. and Ingersoll, A.P. (1996) Bull. Am. Astron. Soc., 28, No. 3, p. 1141.

Sromovsky, L.A. et al. (1996) Science, 272 , pp. 851-853.

von Zahn, U. and Hunten, D.M. (1996) Science, 272, pp. 849-851.

Stevenson, D.J. and Salpeter, E.E. (1977) Astrophys. J. Suppl, 35, p. 221.

Weidenschilling, S.J. and Lewis, J.S. (1973) Icarus, 20, pp. 465-476. 